\title{
Accuracy Verification of Heart Rate and Energy Consumption Tracking Devices to Develop Forest-Based Customized Health Care Service Programs
}

\author{
Jong-Hwan Choi and Hyeon-Ju Kim* \\ Department of Physical Education, Chungbuk National University, Cheongju 28644, Korea
}

\begin{abstract}
This study was carried out to verify the accuracy of fitness tracking devices in monitoring heart rate and energy consumption and to contribute to the development of a forest exercise program that can recommend the intensity and amount of forest exercises based on personal health-related data and provide monitoring and feedback on forest exercises. Among several commercially available wearable devices, Fitbit was selected for the research, as it provides Open API and data collected by Fitbit can be utilized by third parties to develop programs. Fitbit provides users with various information collected during forest exercises including exercise time and distance, heart rate, energy consumption, as well as the altitude and slope of forests collected by GPS. However, in order to verify the usability of the heart rate and energy consumption data collected by Fitbit in forest, the accuracy of heart rate and energy consumption were verified by comparing the data collected by Fitbit and reference. In this study, 13 middleaged women were participated, and it was found that the heart rate measured by Fitbit showed a very low error rate and high correlation with that measured by the reference. The energy consumption measured by Fitbit was not significantly different from that measured in the reference, but the error rate was slightly higher. However, there was high correlation between the results measured by Fibit and the reference, therefore, it can be concluded that Fitbit can be utilized in developing actual forest exercise programs.
\end{abstract}

Keywords: Actigraph, error rate of heart rate, error rate of energy consumption, Fitbit, forest walking

\section{Introduction}

The number of people who visit forests for healthcare has been increasing annually (Korea Forest Service, 2017), and exercises in forests were reported to increase physical strength such as the muscular strength of the lower body, flexibility, agility-balance and cardiorespiratory endurance (Choi et al., 2014; Lee et al., 2016; Choi et al., 2018), and to have a positive impact on physiological variations including the blood sugar of non-insulin dependent diabetes mellitus patients, melatonin, superoxide dismutase (SOD), lipoperoxide, heart rate and blood pressure (Park, 2010; Shin et al., 2011; Lee et al., 2017).

\footnotetext{
This study was carried out with the support of R\&D Program for Forest Science Technology (Project No. 2016004B10-1619-AB01) provided by Korea Forest Service (Korea Forestry Promotion Institute).
}

Received: February 21, 2019, Revised: March 13, 2019, Accepted: April 3, 2019

First author: Jong-Hwan Choi, E-mail: choij@chungbuk.ac.kr, (1) https://orcid.org/0000-0001-7478-0538

*Corresponding author: Hyeon-Ju Kim, E-mail: khju73@cbnu.ac.kr, (1) https://orcid.org/0000-0003-4658-7997 
As people's interest in healthcare is growing, the healthcare market that utilizes various information and communication technology (ICT) and devices has rapidly expanded. However, there has been no study on healthcare service programs that utilize a wide range of ICT in the area of forest therapy, and their effects. In addition, earlier studies that reported that regular exercises in forests improved physical and physiological functions mostly adjusted the intensity of exercises and suggested exercise hours and frequency simply by measuring heart rate, and analyzed their effects (Choi et al., 2018; Choi et al., 2011; Shin et al., 2015; Lee et al., 2017). In order to verify the effects of forest exercises more accurately and identify the relationship between health and death rate by tracking exercises for a long term, data on physical activities need to be collected and connected to networks. In this regard, we have conducted studies with the aim of recommending suitable intensities and amounts of exercises in forests by measuring personal health-related data and developing forest exercise programs that can monitor and provide feedback on actual exercises in forests. As our earlier studies showed, motives for exercising in forests can be provided and scientific basis for forest exercises can be established not simply by showing heart rate during exercises, but also by monitoring forest exercises in real time and providing feedback on whether the recommended intensity and amount of exercises are achieved after exercises, and accumulating data on forest exercises.

Meanwhile, it is essential to use wearable devices in order to monitor forest exercises in real time and provide feedback on whether the recommended intensity and amount of exercises are achieved. Currently, Polar, a device to measure heart rate wirelessly during forest exercises, is widely used (Choi et al., 2018; Choi et al., 2011; Shin et al., 2015; Lee et al., 2017), and it is known as a precise ECG device with very high accuracy (Engström et al., 2012; Gillinov et al., 2017; Hough et al., 2017; Wang et al., 2017). The amount of exercises, that is, energy consumption, during forest exercises have been hardly measured, but energy consumption during general exercises has been widely measured using Actograph's validated 3-axis accelerometer (Sasaki et al., 2011; Lee, 2012; Lee et al., 2014; Lee and Choi, 2015). Respiratory gas analyzers are used as a device to verify the measured energy consumption in order to measure the precise amount of oxygen and energy consumption (Lamonte and Ainsworth, 2001; Schutz et al., 2001). However, since the wireless heart rate measuring device (Polar) and the accelerometer (Actigraph) can collect data in real time, but are not connected to networks, it is difficult to utilize them in application-based customized forest exercise programs.

Various types of wrist wearable products have been developed and utilized. According to a report released by Gartner, an IT research company in the United States (Gartner, 2018), the number of wearable devices shipped in 2019 is predicted to be 225 million units, up by $25.8 \%$ from the number in 2018, and $39 \%$ out of them will be wrist wearable products. Another market report released by International Data Corporation showed that the market share of Mi Band (Xiaomi, China) was the highest in the fourth quarter of 2018, followed by Apple Watch (Apple Inc, USA) and Fitbit (Fitbit Inc, USA) (IDC, 2018). Xiaomi's Mi Band is a very low-end model, and there are not much data available on the model. Apple Watch offers various functions and shows high accuracy of the heart rate measured during physical activities (Hough et al., 2017; Gillinov et al., 2017; Shcherbina et al., 2017; Wang et al., 2017), but despite these advantages the iOS-based product is only for iPhone users, not available for Android phone users. Meanwhile, Fitbit is a hybrid application both for iOS and Android-based devices, and has other advantages such as reasonable price and open API that allows other programs developed by third parties to utilize data collected by Fitbit in the programs. In other words, a wide range of data collected during forest exercises such as exercise time and distance, heart rate and energy consumption, and also data collected by GPS such as forest altitude and slope can be utilized in developing application-based customized forest exercise programs. The accuracy of the heart rate measured during physical activities by Fitbit is high (Hough et al., 2017; Shcherbina et al., 2017; Wang et al., 2017), but it was reported that the error rate of energy consumption was very high (Lee et al., 2014; Thiebaud et al., 2018; Shcherbina et al., 2017).

These studies on the accuracy of heart rate and energy consumption were conducted in a laboratory environment using 
a treadmill or bicycle ergometer and collected data for 3-10 minutes at different exercise intensities, but there was no study that analyzed the accuracy of data measured while walking in actual forests. In particular, forests have different topographical elements such as uneven ground, obstacles like rocks, trees and leaves, and uphill and downhill trails, and other factors such as uneven strides and exercise speed are involved. For this reason, data on the accuracy of heart rate and energy consumption while walking in forests using Fitbit are expected to be utilized as basic data in developing customized forest exercise programs in the future.

Against this backdrop, the heart rate and energy consumption of middle-aged women who showed the highest participation rate in forest exercises (Cho et al., 2014; Park, 2014) were monitored while walking in forest to compare the collected data with those collected using the reference devices, and thus to verify testing devices' accuracy.

\section{Research Methods}

\section{Subjects}

The subjects of this study were selected among those who had ever attended classes offered by a university for local residents, and 13 middle-aged women (55.92 \pm 3.07 years old on average) who listened to explanations about this study and voluntarily expressed consent to participate were selected (Table 1). Subjects were selected among healthy adults who did not have any special disease that was not allowed to do activities used in this study such as cardiac disorders, mental disorders, cognitive disorders, visual impairment, physical disorders, and were able to participate in activities such as walking in forest. In terms of body mass index (BMI) (American College of Sports Medicine, 2014), One subject was underweight; 11 subjects, normal; and one subject, overweight, and those who were obese were not included in this study. This study was approved by the Institutional Review Board of Chungbuk National University (CBNU-201810-BMSBBR723-01), and every participant was instructed to sign a consent form following the approved procedure. The average time

Table 1. Characteristics of participants $(\mathrm{N}=13)$

\begin{tabular}{|c|c|c|c|c|c|c|}
\hline No & Gender & $\begin{array}{l}\text { Age } \\
\text { (years) }\end{array}$ & $\begin{array}{l}\text { Height } \\
(\mathrm{cm})\end{array}$ & $\begin{array}{l}\text { Weight } \\
(\mathrm{kg})\end{array}$ & $\begin{array}{c}\text { BMI } \\
\left(\mathrm{kg} / \mathrm{m}^{2}\right)\end{array}$ & $\begin{array}{l}\text { Forest walking time } \\
\text { (min) }\end{array}$ \\
\hline 1 & \multirow{13}{*}{ Female } & 49 & 158.9 & 50.2 & 19.88 & 69.47 \\
\hline 2 & & 58 & 158.0 & 55.0 & 22.03 & 61.17 \\
\hline 3 & & 56 & 158.6 & 56.7 & 22.54 & 71.67 \\
\hline 4 & & 53 & 151.0 & 52.7 & 23.11 & 66.28 \\
\hline 5 & & 54 & 159.0 & 45.0 & 17.80 & 68.55 \\
\hline 6 & & 58 & 167.0 & 77.4 & 27.75 & 66.35 \\
\hline 7 & & 58 & 162.6 & 62.7 & 23.72 & 63.15 \\
\hline 8 & & 55 & 161.0 & 55.0 & 21.22 & 60.00 \\
\hline 9 & & 55 & 161.3 & 61.1 & 23.48 & 56.40 \\
\hline 10 & & 62 & 155.4 & 57.4 & 23.77 & 64.95 \\
\hline 11 & & 56 & 161.3 & 61.1 & 23.48 & 61.15 \\
\hline 12 & & 57 & 160.2 & 51.6 & 20.11 & 67.19 \\
\hline 13 & & 56 & 165.5 & 65.0 & 23.73 & 59.65 \\
\hline & \pm SD & $55.92 \pm 3.07$ & $159.98 \pm 4.09$ & $57.76 \pm 8.09$ & $22.51 \pm 2.43$ & $64.31 \pm 4.47$ \\
\hline
\end{tabular}


for subjects to walk the same distance was 64 minutes 19 seconds, and the heart rate and energy consumption of subjects during the period were measured every 30 seconds and 1 minute respectively. Data were collected from a small group of 13 persons in this study, but the number of data about heart rate and energy consumption analyzed in this study was 1682 and 837 respectively. They were used to compare accuracy between data corresponding to each time (Benedetto et al., 2018; Gillinov et al., 2017; Stahl et al., 2016; Wang et al., 2017).

\section{Measuring tools and procedures}

The purpose of this study was to analyze the accuracy of the heart rate and energy consumption measured using a wearable device. The devices used to measure the heart rate and energy consumption of subjects as the recognized reference standard (gold standard) were as follows: Polar Bluetooth Smart (Polar Electro, Finland) for heart rate and K5 (Cosmed, Italy), a wireless respiratory gas analyzer, for energy consumption. The most accurate gold standard method of heart rate measuring is an electrocardiography (ECG) monitor, but Hough et al. (2017), Gillinov et al. (2017), and Wang et al. (2017) reported the correlation of higher than .99 between the heart rates measured by Polar and an ECG monitor. Thus, Polar was used as the gold standard in this study.

The device used to compare the accuracy of the gold standard devices for heart rate and energy consumption was Fitbit Charge 2 (Fitbit Inc., USA), and Actigraph GT3X+ (Actigraph, FL), a 3-axis accelerometer that is widely used to measure energy consumption during exercises both in daily life and in the field of research, was additionally used to measure energy consumption.

All of the subjects gathered to visit the forest course they would walk 3 days prior to the actual test day, and they were instructed not to eat or exercise from 3 hours before the test on the day in order to prevent any additional variability of energy consumption. Waiting at the starting place, they wore a belt-type transmitter on the chest, Fitbit Charge 2 on the left wrist, a watch-type Polar receiver on the right wrist, and Actigraph GT3X+ on the bare skin of the left waist. K5 was calibrated right before the experiment, and the main body of K5 was fixed on a harness. Subjects wore the harness on the back and tightly put on a breathing mask connected to the harness. Once subjects wore every device, the start buttons on Fitbit, Polar and K5 were simultaneously pressed. Subjects were instructed to walk along the designated course, to touch every point that was marked in advance, and to press the end buttons on the three devices after returning to the starting place. Actigraph did not require any manual operation, but automatically measured energy consumption within the pre-set period. The intensity of walking was maintained to keep individuals' $\mathrm{VO}_{2} \mathrm{R}$ within the range of $50-70 \%$.

\section{Geographical features in forest}

The GPS file of forest walking provided by Fitbit was analyzed using the Google Earth Pro program and the results were as shown in Figure 1. The total length of the forest walking course was about 4,581m, and geographical features were divided into (1) uphill path, (2) downhill path and (3) gentle-slope path. The distance and slope degree of each path were listed as follows. The altitude of the starting place was $123 \mathrm{~m}$, and the maximum altitude was $170 \mathrm{~m}$. The average slope of the whole course was within the gentle slope range $\left(5-15^{\circ}\right)$ of forest areas designated by the Ministry of Environment or lower, which is a very easy level for hiking.

\section{Data processing methods}

The heart rate and energy consumption measured using each tool that subjects wore during forest walking were summarized by the unit of 30 seconds and 1 minute respectively. Since Fitbit provides data on energy consumption every 


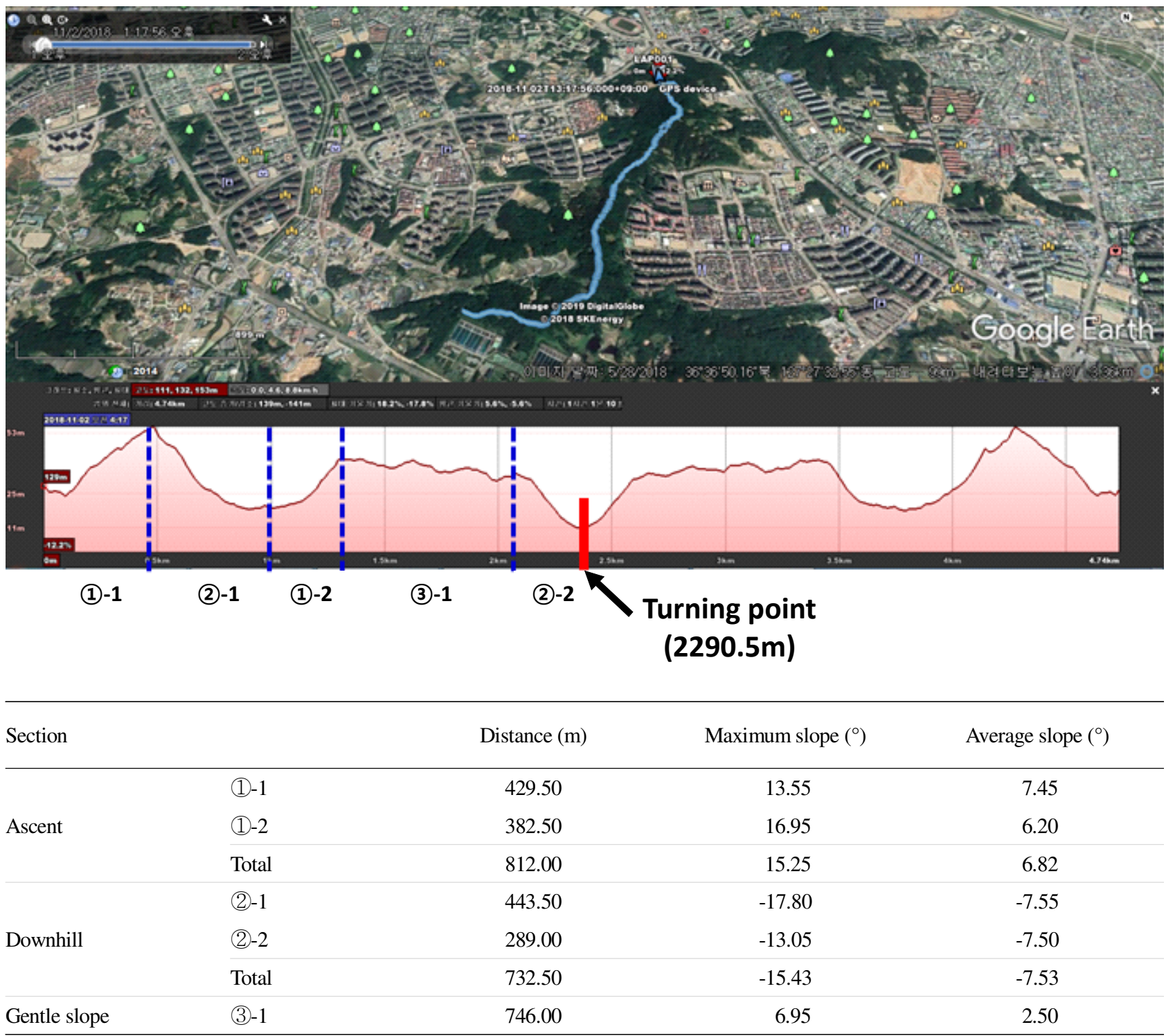

Figure 1. Forest topography measured by GPS of Fitbit.

one minute, the data unit of Actigraph and K5 has to be reset into 1 minute, and the validity of the measured data can be verified by analyzing the correlation and error rate between data corresponding each time. In addition, since basal metabolism is included in energy consumption data provided by Fitbit every one minute, actual energy consumption can be obtained by subtracting basal metabolism from the measured energy consumption.

To analyze the accuracy of the heart rate and energy consumption measured using each tool during the forest exercise, the correlation coefficient (r) between gold standard and testing device data was calculated. Absolute error rate (\%) was calculated using the following equation, [(testing device measurement - gold standard)/gold standard] $\times 100$, and relative errors were also calculated to identify the overestimated or underestimated level of error rates. In addition, significant difference tests (independent t-test, One-way ANOVA) were conducted on the error rate of each tool. All the collected data in this study were processed using the SPSS WIN 20.0 program, and the statistical significance level was set at $\alpha=.05$. 


\section{Results and Discussion}

\section{Accuracy of heart rate measuring tool during forest exercise}

Differences in the heart rate of subjects measured for each segment of geographical features by the gold standard, Polar, and the testing device, Fitbit, were identified and the error rate and correlation between the two were analyzed (Table 2).

There was no statistically significant difference between the heart rate measured by Polar and that measured by Fitbit, and there was also no difference by geographical features. In addition, error rates were analyzed, and the error rate between the heart rate measured by Fitbit and that measured by Polar over the whole course was very low (maximum error, 2.39\%), showing very high correlation ( $\mathrm{r}=.96$ or higher).

These results were similar to those of earlier studies that reported the high correlation between the heart rate measured by Fitbit and that measured by ECG, reference standard, in a laboratory environment composed of a treadmill and bicycle ergometer (Stahl et al, 2016; Hough et al., 2017; Gillinov et al., 2017; Shcherbina et al., 2017; Wang et al., 2017). Stahl et al. (2016) reported that the heart rate measured by Fitbit Charge HR during walking and running on a treadmill showed the correlation of $\mathrm{r}=.93$ with that measured in reference standard, and that the error rate between different intensities of exercises was within $1.73-10.06 \%$. Thiebaud et al. (2018) reported the error rate of 2.17-8.06\%, and concluded that the accuracy of the heart rate measured by Fitbit was high.

Meanwhile, Gillinov et al. (2017) reported that the correlation during running on a treadmill was $r=.76$, but that the correlation during riding a fixed bicycle was very low, showing differences in heart rate depending on the type of physical activities. The error rate was also found to be relatively high (10-15\%), which was slightly different from high correlation and low error rates even in different geographical feasures in this study. Benedetto et al. (2018) reported that the heart rate measured by Fitbit was lower than that measured in reference standard by 5.9 beats, and that the maximum difference was 30 beats, and Wallen et al. (2016) also reported that the heart rate was lower by 2.5-9.3 beats. Stahl et al. (2016) reported that the heart rate was lower than that measured by Polar. These studies pointed out that the algorithm used in Fitbit slightly lacked accuracy. In addition, Alzahrani et al. (2015) reported that measuring the heart rate using Fitbit can be affected by the amount of blood related to the systolic pulse of the heart, the movements of the arms, the adherence of sensors on the skin, the color and brightness of the skin, ambient brightness, bad blood perfusion, etc., indicating that these factors might cause differences in the accuracy of the heart rate measured by Fitbit. For this reason, it will be necessary to conduct an additional study on the accuracy of the heart rate targeting not only females but also males, different age groups and those with different health conditions during different physical activities under different intensities.

Still, the results of many earlier studies and this study that examined the accuracy of the measured heart rate indicated

Table 2. Differences in heart rate monitoring, error rates in Fitbit compared to Polar reference, and correlation between them in forest walking

\begin{tabular}{|c|c|c|c|c|c|c|c|c|}
\hline Slope & $\begin{array}{c}\text { Polar } \\
(\mathrm{M} \pm \mathrm{SD})\end{array}$ & $\begin{array}{c}\text { Fitbit } \\
(\mathrm{M} \pm \mathrm{SD})\end{array}$ & $\mathrm{t}$ & Device & Slope & $\begin{array}{c}\text { MAPE } \\
(\%)\end{array}$ & $\begin{array}{c}\text { MPE } \\
(\%)\end{array}$ & Correlation \\
\hline Ascent & $132.13 \pm 17.86$ & $130.57 \pm 17.30$ & -0.567 & \multirow{4}{*}{ Fitbit } & Ascent & $2.39 \pm 2.69$ & $1.07 \pm 3.44$ & $.97^{* * *}$ \\
\hline Downhill & $121.45 \pm 13.61$ & $120.62 \pm 13.50$ & -1.645 & & Downhill & $2.24 \pm 5.56$ & $0.63 \pm 3.35$ & $.96^{* * *}$ \\
\hline Gentle slope & $124.83 \pm 13.04$ & $125.52 \pm 15.60$ & -1.307 & & Gentle slope & $1.89 \pm 1.81$ & $0.45 \pm 2.58$ & $.97^{* * *}$ \\
\hline Total & $126.59 \pm 16.05$ & $125.52 \pm 15.60$ & -1.961 & & Total & $2.22 \pm 2.46$ & $0.76 \pm 3.22$ & $.97^{* * * *}$ \\
\hline
\end{tabular}

Note MAPE = mean absolute percent error; $\mathrm{MPE}=$ mean percent error. MAPE and MPE values are mean and standard deviation. ${ }^{* * * *} p<.001$. 
that Fitbit is a very useful tool to monitor the heart rate of middle-aged women during forest walking. The high accuracy of the heart rate measured by Fitbit in this study demonstrates the high potential of Fitbit for being utilized in developing forest exercise programs, and the possibility of utilizing it in developing forest exercise programs. A proper intensity (heart rate) of exercises can be recommended based on the actual physical strength, diseases and health conditions of users, and monitoring whether they achieved the goal or not and giving feedback will be very helpful for users to do effective forest exercises.

\section{Accuracy of energy consumption measuring tool}

The energy consumption measured by K5, gold standard for monitoring energy consumption, and those measured by Fitbit and Actigraph by forest geographical features were compared, and the results and error rates were listed as follows (Tables 3 and 4).

Differences between three devices by forest geographical feature were analyzed (Table 3), and there were significant differences between the energy consumption measured by K5 and that measured by Actigraph in all of the geographical features. However, there was no significant difference between Fitbit and K5, gold standard. In addition, the error rates of both Fitbit (15.17-18.81\%) and Actigraph (21.42-35.60\%) were slightly high. In the case of Actigraph, a higher error rate in energy consumption measurement compared to the reference was observed. Error rates depending on the type of geo-

Table 3. Differences in energy consumption measurements and post hoc analysis between devices and $\mathrm{K} 5$ reference in forest walking

\begin{tabular}{lccccc}
\hline Slope & $\begin{array}{c}\mathrm{K} 5 \\
(\mathrm{M} \pm \mathrm{SD})\end{array}$ & $\begin{array}{c}\text { Fitbit } \\
(\mathrm{M} \pm \mathrm{SD})\end{array}$ & $\begin{array}{c}\text { Actigraph } \\
(\mathrm{M} \pm \mathrm{SD})\end{array}$ & $\mathrm{F}$ & Post-hoc $^{\mathrm{z}}$ \\
\hline Ascent & $136.92 \pm 21.31$ & $132.68 \pm 20.94$ & $90.60 \pm 20.43$ & $202.61^{* * * *}$ & $3<2,1$ \\
Downhill & $94.15 \pm 19.38$ & $90.69 \pm 24.04$ & $88.23 \pm 24.35$ & $4.68^{*}$ & $3<1$ \\
Gentle slope & $76.85 \pm 14.95$ & $76.79 \pm 17.49$ & $66.49 \pm 13.53$ & $28.81^{* * *}$ & $3<2,1$ \\
Total & $307.92 \pm 41.32$ & $300.17 \pm 49.31$ & $245.32 \pm 51.34$ & $165.25^{* * *}$ & $3<2,1$ \\
\hline
\end{tabular}

${ }^{\mathrm{z}} 1=\mathrm{K} 5 ; 2=$ Fitbit; $3=$ Actigraph.

${ }^{*} p<.05, \stackrel{* * * *}{p}<.001$.

Table 4. Error rates of devices in energy consumption measurements compared to $\mathrm{K} 5$ reference and post hoc analysis in forest walking

\begin{tabular}{|c|c|c|c|c|c|}
\hline Device & Slope & $\begin{array}{l}\text { MAPE } \\
(\%)\end{array}$ & $\begin{array}{c}\mathrm{F} \\
\text { (by slope) }\end{array}$ & Post-hoc ${ }^{z}$ & $\begin{array}{l}\text { MPE } \\
(\%)\end{array}$ \\
\hline \multirow{4}{*}{ Fitbit } & Ascent & $15.17 \pm 13.15$ & \multirow{4}{*}{$5.34^{* *}$} & \multirow{4}{*}{$1<2$} & $2.17 \pm 19.98$ \\
\hline & Downhill & $18.81 \pm 14.42$ & & & $1.23 \pm 23.77$ \\
\hline & Gentle slope & $17.19 \pm 17.26$ & & & $-1.90 \pm 24.30$ \\
\hline & Total & $16.99 \pm 14.80$ & & & $0.80 \pm 22.52$ \\
\hline \multirow{4}{*}{ Actigraph } & Ascent & $35.60 \pm 17.00$ & \multirow{4}{*}{$51.87^{* *}$} & \multirow{4}{*}{$3,2<1$} & $29.60 \pm 26.10$ \\
\hline & Downhill & $24.24 \pm 20.10$ & & & $1.84 \pm 31.62$ \\
\hline & Gentle slope & $21.42 \pm 15.02$ & & & $10.51 \pm 24.17$ \\
\hline & Total & $28.01 \pm 18.74$ & & & $15.14 \pm 30.26$ \\
\hline
\end{tabular}

Note. $\mathrm{MAPE}=$ mean absolute percent error; MPE $=$ mean percent error. MAPE and MPE values are mean and standard deviation.

${ }^{\mathrm{z}} 1=\mathrm{K} 5 ; 2=$ Fitbit; 3 = Actigraph.

*** $p<.01$. 
graphical features were additionally analyzed. Fitbit showed a higher error rate in the downhill path, while Actigraph showed a higher error rate in the uphill path (Table 4).

Meanwhile, the correlation between energy consumption measured by K5, gold standard, and that measured by Fitbit and Actigraph was analyzed (Table 5). The energy consumption measured by Fitbit showed a close correlation with that measured by $\mathrm{K} 5$ in the reference test $(\mathrm{r}=.72)$, but Actigraph did not show any significant correlation.

The results of this study showed that there was no significant difference between energy consumption measured by Fitbit and that measured by K5 reference, but had high correlation, indicating that Fitbit can be used to measure energy consumption during forest exercises. These results highly correspond to those of Lee et al. (2014). The study also reported that the data measured by Fitbit showed high correlation with those measured by reference, and thus that Fitbit can be utilized to measure energy consumption, but reported the error rate of $10 \%$. Energy consumption, that is whether to achieve exercise goals, has a very big impact on the effect of exercises. In fact, the results of the meta analysis of the effect of exercise programs for obese adults showed that the exercise program that suggested the amount of exercise (energy consumption) was more effective in addressing obesity than the other program that simply suggested the time of exercise and heart rate (Kim and Choi, 2017), indicating that energy consumption is a very important factor in effective exercise programs.

Chowdhury et al. (2017), however, reported that Fitbit showed different error rates of energy consumption depending on the type of physical activities. Walking up and down stairs and running showed the error rate of $15-16 \%$, while walking on a treadmill and cycling showed a high error rate (75\% and 53\% respectively). The average error rate of the whole physical activities was 36\%. The average error rate of Apple Watch was 27\%, and that of Microsoft Band was 40\%, which indicates that wearable devices show a low accuracy in terms of energy consumption. When measuring energy consumption in daily life for 24 hours, that measured by Fitbit and Apple Watch was $405 \mathrm{kcal}$ and $394 \mathrm{kcal}$, showing underestimated results. The energy consumption measured by Microsoft band that uses multi-sensors like Fitbit and Apple Watch, and Jawbone UP24 that uses an accelerometer was underestimated by approximately 1,000 kcal. Meanwhile, Actiheart (Cambridge Neurotechnology Ltd, UK) is used in the field of research to accurately measure energy consumption in daily life through the doubly labeled water method and validity verification (Nightingale et al., 2015; The InterAct Consortium, 2012), and BodyMedia Core armband (BodyMedia Inc., PA) is used in the field of research to quantify energy consumption through accuracy verification with reference (Bond et al., 2014; Peacock et al., 2015). The two tools also showed the error rate of $20 \%$ and $33 \%$ respectively on average in different physical activities, and the energy consumption measured by the two tools for 24 hours also showed similar results to those of Apple and Fitbit devices (Chowdhury et al., 2017). In other words, energy consumption measured not only by wearable devices but also by research tools during physical activities and in daily life showed a high error rate. Shcherbina et al. (2017) and Thiebaud et al. (2018) also reported that Fitbit showed high accuracy in measuring heart rate, but that it showed a high error rate in measuring energy consumption. These studies pointed out that energy consumption needs to be estimated by comprehensively considering height, weight and exercise intensity, that it is still difficult to accurately measure energy consumption with the existing algorithm, and thus that it needs to be carefully applied to patients or groups of people whose exercises need to be controlled in detail.

Table 5. Correlation between energy consumption measurements by devices and $\mathrm{K} 5$ reference in forest walking

\begin{tabular}{lccc}
\hline & K5 & Fitbit & Actigraph \\
\hline K5 & 1 & .72 & .22 \\
Fitbit & - & 1 & .29 \\
\hline
\end{tabular}


These results demonstrate that Fitbit is suitable to measure energy consumption during forest walking when comparing it with the results of the reference, but that it still shows a certain level of error rate. Despite these errors in the current level of technology, wearable devices provide users with feedback in real time (Patel et al., 2015), and personalized data on physical activities (Western et al., 2015), and thus are expected to effectively induce changes in behaviors (Chowdhury et. al., 2017). Therefore, it will be better to recommend a safe range of the amount of exercises with the error rate into consideration instead of recommending a precise amount of exercises based on energy consumption in developing an actual forest exercise program. In general, the recommended amount of exercises per week in order to maintain health is no less than $1,000 \mathrm{kcal}$, and it is recommended to consume 2,000 kcal by doing exercises 7 days a week in order to actively address obesity and diabetes (American College of Sports Medicine, 2014).

Meanwhile, some unexpected results were obtained in this study. Many earlier studies reported that Actigraph, an 3-axis accelerometer, was a suitable device to measure energy consumption, and in fact it has been widely used in the field of research (Lyden et al., 2011; Sasaki et al., 2011; Lee, 2012; Lee et al., 2014; Lee and Choi, 2015). The device is much more expensive than smart watches, but in this study Actigraph was found not to be suitable to measure energy consumption during forest exercises. It also showed a significant difference from the energy consumption measured by the reference, K5, did not show any significant correlation, and showed a higher error rate than that of Fibit. In other words, these different results can be attributed to the fact that movements in an laboratory environment are significantly different from those in an actual forest environment. From this perspective, Fitbit's high correlation with the results of the reference in a forest environment that has uneven ground and various obstacles demonstrates that Fitbit can be utilized as a useful tool to monitor forest exercises.

\section{Conclusion}

This study compared and verified the accuracy of the heart rate and energy consumption measured by Fitbit, a wrist wearable device, and those measured by reference device in order to identify the possibility of utilizing Fitbit to develop forest exercise programs and ultimately forest-based customized healthcare service programs. Fitbit showed a relatively low error rate and high correlation in the heart rate with Polar, gold standard, and the similar results were observed in every geographical feature in a forest that has uphill, downhill and gentle-slope paths, indicating that Fitbit is a useful tool in monitoring the heart rate of middle-aged women during forest walking. In addition, the accuracy of energy consumption measured by Fitbit was analyzed, and there was no significant difference, but a high correlation between Fitbit and K5, a wireless respiratory gas analyzer used as gold standard in this study, indicating that Fitbit can be used to assess energy consumption during forest exercises although its error rate was slightly high. High error rates are also observed in other wearable devices and research devices. Considering the current level of technology, it will be better to recommend a slightly rough range of the amount of exercises instead of a precise amount of exercises based on energy consumption during forest exercises.

Therefore, Fitbit seems to be a useful tool that can be utilized in monitoring exercises in a forest environment that has uneven ground and various obstacles. It will be possible to develop forest-based customized healthcare service programs that can recommend a proper amount of exercises based on individuals' health conditions, and monitor their heart rate and energy consumption through wrist wearable devices, which is expected to provide science-based forest exercises and motives for continuously doing forest exercises. Data accumulated from the use of these services can be utilized to recommend customized forest therapy programs, analyze the effects of forest therapy, treat diseases and manage health conditions. 


\section{References}

Alzahrani, A., S. Hu, V. Azorin-Peris, L. Barret, D. Esliger, M. Hayes, S. Akbare, J. Achart, and S. Kuoch. 2015. A multichannel opto-electronic sensor to accurately monitor heart rate against motion artefact during exercise. Sensors(Basel) 15(10):25681-25702. https://doi.org/10.3390/s151025681

American College of Sports Medicine. 2014. ACSM's guidelines for exercise testing and prescription(9th ed.). Baltimore, MD: Lippincott Williams \& Wilkins.

Benedetto, S., C. Caldato, E. Bazzan, D.C. Greenwood, V. Pensabene, and P. Actis. 2018. Assessment of the Fitbit Charge 2 for monitoring heart rate. PLoS One 13(2):e0192691. https://doi.org/10.1371/journal.pone.0192691

Bond, D.S., J.G. Thomas, H.A. Raynor, J. Moon, J. Sieling, J. Trautvetter, T. Leblond, and R.R. Wing. 2014. B-MOBILE - a smartphone-based intervention to reduce sedentary time in overweight/obese individuals: A within-subjects experimental trial. PLoS One 9(6):e100821. https://doi.org/10.1371/journal.pone.0100821

Cho, T., Y. Lee, and S.M. Kim. 2014. Forest therapy participation and its economic spillover effects. J. Ind. Econ. Bus. 27(4):1479-1499. Retrieved from http://www.kiea.ne.kr

Choi, J., C.S. Shin, and P.S. Yeoun. 2014. Effects of forest-walking exercise on functional fitness and gait pattern in the elderly. J. Korean For. Soc. 103(3):503-509. https://doi.org/10.14578/jkfs.2014.103.3.503

Choi, J., S.R. Yoo, and H.J. Kim. 2018. The effects of forest exercise program on health-related fitness and functional movements in 50s females. Asia Pac. J. Converg. Res. Interchange. 8(7):567-577. https://doi.org/10.21742/AJMAHS.2018.07.31

Choi, K.M., W.S. Shin, P.S. Yeoun, and Y.M. Cho. 2011. The influence of forest walking exercise on human, stress and fatigue. J. Korean Inst. For. Recreat. 15(1):61-66.

Chowdhury, E.A., M.J. Western, T.E. Nightingale, O.J. Peacock, and D. Thompson. 2017. Assessment of laboratory and daily energy expenditure estimates from consumer multi-sensor physical activity monitors. PLoS One 12(2): e0171720. https://doi.org/10.1371/journal.pone.0171720

Engström, E., E. Ottosson, B. Wohlfart, N. Grundström, and A. Wisén. 2012. Comparison of heart rate measured by Polar RS400 and ECG, validity and repeatability. Adv. Physiother. 14(3):115-122. https://doi.org/10.3109/14038196.2012.694118

Gartner. 2018. Gartner says worldwide wearable device sales to grow 26 percent in 2019. Retrieved from https://www.gartner.com/en/newsroom/press-releases/2018-11-29-gartner-says-worldwide-wearable-device-sales-to-grow-

Gillinov, S., M. Etiwy, R. Wang, G. Blackburn, D. Phelan, A.M. Gillinov, P.J. Houghtaling, H. Javadikasgari, and M. Desai. 2017. Variable accuracy of wearable heart rate monitors during aerobic exercise. Med. Sci. Sports Exerc. 49(8):1697-1703. https://doi.org/10.1249/MSS.0000000000001284

Hough, P., M. Glaister, and A. Pledger. 2017. The accuracy of wrist-worn heart rate monitors across a range of exercise intensities. J. Phys. Act. Res. 2(2):112-116. https://doi.org/10.12691/jpar-2-2-8

IDC. 2018, December 3. New product launches drive double-digit growth in the wearables market, says IDC. Retrieved from https://www.idc.com/getdoc.jsp?containerId=prUS44500418

Kim, J. and J. Choi. 2017. Analysis of effect size of aerobic exercise program based on calorie consumption on decreasing body fat percentage of obese patients. Korean J. Sports Sci. 26(6):1203-1220.

Korea Forest Service. 2017. Forest welfare promotion plan. Retrieved from http://www.forest.go.kr

Lamonte, M.J. and B.E. Ainsworth. 2001. Quantifying energy expenditure and physical activity in the context of dose response. Med. Sci. Sports Exerc. 33(6 Suppl):S370-378. https://doi.org/10.1097/00005768-200106001-00006

Lee, J., H. An, S.K. Kang, Y. Kim, and D. Dinkel. 2016. Examining the validity of Fitbit charger HR for measuring heart rate in free-living conditions: 2792 Board \#315 June 3, 9:30AM-11:00AM. Med. Sci. Sports Exerc. 48(5S Suppl 1): 786-787. https://doi.org/10.1249/01.mss.0000487361.48518.aa

Lee, J., Y. Kim, and G.J. Welk. 2014. Validity of Consumer-Based Physical Activity Monitors. Med. Sci. Sports Exerc. 46(9): 1840-1848. https://doi.org/10.1249/MSS.0000000000000287

Lee, M. 2012. Criterion and convergent validity evidences of an accelerometer (Actigraph GT3X) and a pedometer (Omron HJ720IT). Korean J. Meas. Eval. Phys. Educ. Sports Sci. 14(2):1-13. 
Lee, M., C.S. Shin, P.S. Yeon, M.J. Shin, J.S. Lee, and J.H. Choi. 2017. The effects of forest-walking exercise on NK cells and blood melatonin levels of women in their 50s. J. Korean Inst. For. Recreat. 21(2):39-52.

Lee, M. and J. Choi. 2015. Accuracy of wearable devices to estimate physical activity level. Korean J. Meas. Eval. Phys. Educ. Sports Sci. 17(2):49-60.

Lyden, K., S.L. Kozey, J.W. Staudenmeyer, and P.S. Freedson. 2011. A comprehensive evaluation of commonly used accelerometer energy expenditure and MET prediction equations. Eur. J Appl. Physiol. 112(2):187-201. https://doi.org/10.1007/s00421-010-1639-8

Nightingale, T.E., J.P. Walhin, D. Thompson, and J.L. Bilzon. 2015. Predicting physical activity energy expenditure in wheelchair users with a multisensor device. BMJ Open Sport Exerc. Med. 1(1). https://doi.org/10.1136/bmjsem-2015-000008

Park, H. 2014. A study on the improvement method and efficient management of healing forest. Master's thesis, Wonkwang University, Iksan, Korea.

Park, K.Y. 2010. Effect of 10-week forest exercise on change of melatonin. Master's thesis, Chungbuk National University, Cheongju, Korea.

Patel, M.S., D.A. Asch, and K.G. Volpp. 2015. Wearable devices as facilitators, not driver, of health behavior change. JAMA 313(5):459-460. https://doi.org/10.1001/jama.2014.14781

Peacock, O.J., M.J. Western, A.M. Batterham, A.Stathi, M. Standage, A. Tapp., P. Bennett, and D. Thompson. 2015. Multidimensional individualised Physical ACTivity (Mi-PACT)--a technology-enabled intervention to promote physical activity in primary care: study protocol for a randomised controlled trial. Trials 16:381.

https://doi.org/10.1186/s13063-015-0892-x

Sasaki, J.E., D. John, and P.S. Freedson. 2011. Validation and comparison of ActiGraph activity monitors. J. Sci. Med. Sport 14(5):411-416. https://doi.org/10.1016/j.jsams.2011.04.003

Schutz, Y., R.L. Weinsier, and G.R. Hunter. 2001. Assessment of free-living physical activity in humans: an overview of currently available and proposed new measures. Obesity 9(6):368-379. https://doi.org/10.1038/oby.2001.48

Shcherbina, A., C.M. Mattsson, D. Waggott, H. Salisbury, J.W. Christle, T. Hastie, M.T. Wheeler, and E.A. Ashley. 2017. Accuracy in wrist-worn, sensor-based measurements of heart rate and energy expenditure in a diverse cohort. J. Pers. Med. 7(2):3. https://doi.org/10.3390/jpm7020003

Shin, M.S., C.S. Shin, M.O. Lee, J.S. Lee, A.R. Choi, and J.H. Choi. 2015. Effects of exercise program with different forest slope on BDNF and depression of women in 50s. J. Korean Inst. For. Recreat. 19(1):109-115.

Shin, W.S., C.S. Shin, P.S. Yeoun, and J.J. Kim. 2011. The influence of interaction with forest on cognitive function. Scand. J. For. Res. 26(6):595-598. https://doi.org/10.1080/02827581.2011.585996

Stahl, S.E., H. An, D.M. Dinkel, J.M. Noble, and J. Lee. 2016. How accurate are the wrist-based heart rate monitor during walking and running activities? Are they accurate enough? BMJ Open Sport Exerc. Med. 2:e000106.

https://doi.org/10.1136/bmjsem-2015-000106

The InterAct Consortium. 2012. Validity of a short questionnaire to assess physical activity in 10 European countries. Eur. J. Epidemiol. 27(1):15-25. https://doi.org/10.1007/s10654-011-9625-y

Thiehaud, R.S., M.D. Funk, J.C. Patton, B.L. Massey, T.E. Shay, M.G. Schmidt, and N. Giovannitti. 2018. Validity of wrist-worn consumer products to measure heart rate and energy expenditure. Digit. Health 4:1-7. https://doi.org/10.1177/2055207618770322

Wallen, M.P., S.R. Gomersall, S.E. Keating, U. Wisløff, and J.S. Coombes. 2016. Accuracy of heart rate watches: Implication for weight management. Plos One 11(5):e0154420. https://doi.org/10.1371/journal.pone.0154420

Wang, R., G. Blackburn, M. Desa.i, D. Phelan, L. Gillinov, P. Houghtaling, and M. Gillinov. 2017. Accuracy of wrist-worn heart rate monitors. JAMA Cardiol. 2(1):104-106. https://doi.org/10.1001/jamacardio.2016.3340

Western, M.J., O.J. Peacock, A. Stathi, and D. Thompson. 2015. The understanding and interpretation of innovative technologyenabled multidimensional physical activity feedback in patients at risk of future chronic disease. PLoS One 10(5): e0126156. https://doi.org/10.1371/journal.pone.0126156 